\title{
LINEAR-QUADRATIC STOCHASTIC DIFFERENTIAL GAMES FOR DISTRIBUTED PARAMETER SYSTEMS
}

\author{
S.-I. Aihara and A. BaGCHI \\ Department of Applied Mathematics, Twente University of Technology, P.O. Box 217. \\ 7500 AE Enschede. The Netherlands
}

\begin{abstract}
A linear-quadratic differential game with infinite dimensional state space is considered. The system state is affected by disturbance and both players have access to different measurements. Optimal linear strategies for the pursuer and the evader, when they exist, are explicitly determined.
\end{abstract}

\section{INTRODUCTION}

We consider a two-person pursuit-evasion differential game with infinite dimensional state space in which both the system state and the measurements are corrupted by noises. The feature that the players have access to different noisy measurements makes the problem already hard for the corresponding lumped-parameter case. This was solved directly in Bagchi and Olsder [1] by introducing new state variables with values in Hilbert space and converting the original problem to an optimization problem with an infinite dimensional state space. Other approaches to the problem may be found in [2] and [3] and numerical aspects are discussed in [4]. We extend here the method proposed in [1] to solve the two-person stochastic pursuit-evasion differential game for distributed parameter systems.

Section 2 starts with some basic formalisms used here on linear partial differential equations and formulates the problem in this framework. In the class of linear strategies, the problem is reformulated in Section 3 into an optimization problem in a different function space. This is solved in Section 4 and representations of the control gain operators arising in the solutions are given in Section 5. The conventional Brownian motion model is used, as opposed to the finitely additive white noise model used in [1].

\section{MATHEMATICAL PRELIMINARIES AND PROBLEM FORMULATION}

Let $V$ and $H$ be two Hilbert spaces, $V \subset H, V$ dense in $H$; let $\|\cdot\|$ and $|\cdot|$ denote the norms in $V$ and $H$ and $(\cdot, \cdot)$ the scalar product in $H$. We identify $H$ with its antidual. With $V^{\prime}$ denoting the antidual of $V$,

$$
V \subset H \subset V^{\prime}
$$

where we assume that the injection of $V$ into $H$ is compact.

Let $-A \in \angle\left(V ; V^{\prime}\right)$ be an operator satisfying

$$
\text { (A-1) }-\langle A \phi, \phi\rangle \geqslant \alpha_{1}\|\phi\|^{2} \text { and }\langle A \phi, \phi\rangle \leqslant \alpha_{2}\|\phi\|^{2} \quad \forall \phi \in V \text {, }
$$

with

$$
x_{1}>0, \quad x_{2}>0
$$

where $\langle\cdot, \cdot\rangle$ denotes the duality between $V$ and $V^{\prime} .(\Omega, \mathscr{B}, P)$ is a complete probability space and $T$ denotes the time interval $\left(0, t_{f}\right)$. Let $H_{n}$ be another Hilbert Space and $W(t)$ be a Wiener process in $H_{n}$ with covariance operator $\mathscr{W}^{\prime}$ nuclear. We consider the following equation describing the evolution of the system in state space:

$$
(x(t), \phi)-\left(x_{0}, \phi\right)=\int_{0}^{t}\langle A x(\tau), \phi\rangle \mathrm{d} \tau+\int_{0}^{t}\left(B_{\mathrm{p}} u_{\mathrm{p}}(\tau)+B_{\mathrm{e}} u_{\mathrm{c}}(\tau), \phi\right) \mathrm{d} \tau+(F W(t), \phi) \quad \forall \phi \in V
$$

where $u_{\mathrm{p}}$ and $u_{\mathrm{e}}$ are the strategies of the pursuer and the evader, respectively, and they belong to 
$L^{2}\left(\Omega \times T ; H_{u}\right), H_{u}$ another separable Hilbert space; $B_{\mathrm{p}}, B_{\mathrm{c}} \in L\left(H_{u} ; H\right)$ are Hilbert-Schmidt and $F \in L\left(H_{n} ; H\right)$. Without loss of generality, we assume that $x_{0} \sim N\left(\bar{x}_{0}, P_{0}\right), \bar{x}_{0}=0$.

The observations available to the pursuer and the evader are, respectively,

$$
\begin{aligned}
& Y_{\mathrm{p}}(t)=\int_{0}^{t} C_{\mathrm{p}} x(\tau) \mathrm{d} \tau+\int_{0}^{t} G_{\mathrm{p}} \mathrm{d} V_{\mathrm{p}}(\tau) \\
& Y_{\mathrm{e}}(t)=\int_{0}^{1} C_{\mathrm{e}} x(\tau) \mathrm{d} \tau+\int_{0}^{t} G_{\mathrm{e}} \mathrm{d} V_{\mathrm{e}}(\tau)
\end{aligned}
$$

where $V_{\mathrm{p}}(t)$ and $V_{\mathrm{c}}(t)$ are vector-valued independent Wiener processes on $\mathbb{R}^{m_{\mathrm{p}}}$ and $\mathbb{R}^{m_{\mathrm{e}}}$, respectively, with covariance matrices $\mathscr{V}_{\mathrm{p}}$ and $\mathscr{V}_{\mathrm{c}} ; \mathscr{V}_{\mathrm{p}}, \mathscr{V}_{\mathrm{p}}^{-1}, G_{\mathrm{p}}, G_{\mathrm{p}}^{-1} \in \angle\left(\mathbb{R}^{m_{\mathrm{p}}}\right) ; \mathscr{V}_{\mathrm{c}}, \mathscr{V}_{\mathrm{c}}^{-1}, G_{\mathrm{c}}, G_{\mathrm{c}}^{-1} \in \angle\left(\mathbb{R}^{m_{\mathrm{c}}}\right)$; $C_{\mathrm{p}} \in L\left(H ; \mathbb{R}^{m_{\mathrm{p}}}\right)$ and $C_{\mathrm{c}} \in \angle\left(H ; \mathbb{R}^{m_{\mathrm{c}}}\right)$. Under the preceding assumptions, one can prove that

$$
x \in L^{2}\left(\Omega ; C(\bar{T} ; H) \cap L^{2}(T ; V)\right), Y_{\mathrm{p}} \in L^{2}\left(\Omega ; C\left(\bar{T} ; \mathbb{R}^{m_{\mathrm{p}}}\right), Y_{\mathrm{e}} \in L^{2}\left(\Omega ; C\left(\bar{T} ; \mathbb{R}^{m_{\mathrm{e}}}\right) .\right.\right.
$$

We assume, from now on, that the strategies $u_{\mathrm{p}}(t)$ and $u_{\mathrm{c}}(t)$, for fixed $t$, are linear functionals of the observations $Y_{\mathrm{p}}(s), 0 \leqslant s \leqslant t$, and $Y_{\mathrm{e}}(s), 0 \leqslant s \leqslant t$, respectively:

$$
\begin{aligned}
& u_{\mathrm{p}}(t)=\int_{0}^{t} N_{\mathrm{p}}(t, \tau) \mathrm{d} Y_{\mathrm{p}}(\tau) \\
& u_{\mathrm{c}}(t)=\int_{0}^{t} N_{\mathrm{c}}(t, \tau) \mathrm{d} Y_{\mathrm{c}}(\tau)
\end{aligned}
$$

where, for each $\tau \leqslant t, N_{\mathrm{p}}(t, \tau) \in \angle\left(\mathbb{R}^{m_{\mathrm{p}}} ; H_{u}\right), N_{\mathrm{c}}(t, \tau) \in \angle\left(\mathbb{R}^{m_{\mathrm{e}}} ; H_{u}\right), \partial N_{\mathrm{p}}(t, \cdot) / \partial \tau \in L^{2}\left(\bar{T} ; \angle\left(\mathbb{R}^{m_{\mathrm{p}}} ; H_{u}\right)\right.$ and $\partial N_{\mathrm{c}}(t, \cdot) / \partial \tau \in L^{2}\left(\bar{T} ;\left(\mathbb{R}^{m_{\mathrm{c}}} ; H_{u}\right)\right)$. Within this class of control strategies, we want to determine the minimax solution

$$
\sup _{u_{\mathrm{c}}} \inf _{u_{\mathrm{p}}} E\{J\}
$$

where

$$
J=\frac{1}{2} \int_{0}^{t_{t}}\left\{|Q x(t)|^{2}+\left|R_{\mathrm{p}} u_{\mathrm{p}}(t)\right|_{H_{u}}^{2}-\left|R_{\mathrm{e}} u_{\mathrm{c}}(t)\right|_{H_{u}}^{2}\right\} \mathrm{d} t
$$

with $R_{\mathrm{p}}, R_{\mathrm{e}}$ positive definite operators in $\angle\left(H_{\mathrm{u}} ; H_{\mathrm{u}}\right\}$ and $Q$ a nonnegative definite Hilbert-Schmidt operator from $H$ into itself.

We assume throughout that both players know and have perfect recall as to the system characteristics:

$$
\left\{A, B_{\mathrm{p}}, B_{\mathrm{e}}, C_{\mathrm{p}}, C_{\mathrm{c}}, F, G_{\mathrm{p}}, G_{\mathrm{c}}, \bar{x}_{0}, P_{0}, \mathscr{W}, \mathscr{Y}_{\mathrm{p}}, \mathscr{Y}_{\mathrm{c}}, Q, R_{\mathrm{p}}, R_{\mathrm{c}}\right\}
$$

and also as to their own past measurements and controls.

\section{REFORMULATION IN A DIFFERENT STATE SPACE}

Let us introduce new state variables

$$
\begin{aligned}
& \pi(t)(s) \triangleq x(t \wedge s) \\
& \eta_{\mathrm{p}}(t)(s) \triangleq \int_{0}^{1 \wedge s} G_{\mathrm{p}} \mathrm{d} V_{\mathrm{p}}, \quad \eta_{\mathrm{e}}(t)(s) \triangleq \int_{0}^{t \wedge s} G_{\mathrm{e}} \mathrm{d} V_{\mathrm{e}}
\end{aligned}
$$

where $t \wedge s \triangleq \min (t, s)$. We convert our original problem into two optimal control problems. First assume that $N_{\mathrm{c}}(t, \tau)$ is fixed and determine the optimal $u_{\mathrm{p}}(t)$ which, of course, will be expressed in terms of $N_{\mathrm{c}}(t, \tau)$. The form of $u_{\mathrm{p}}(t)$ given by (4a) will then express $N_{\mathrm{p}}$ as a function of $N_{\mathrm{c}}$. Interchanging the roles of $u_{\mathrm{p}}$ and $u_{\mathrm{c}}$, we obtain two implicit functional relations for the two unknowns $N_{\mathrm{p}}$ and $N_{\mathrm{e}}$. If this pair of equations has a unique solution, there exists a unique saddle-point of the pursuit-evasion game formulated in Section 2 in the class of strategies given by $(4 a, b)$. Let us now proceed with the above-mentioned scheme. 
Suppose that $N_{\mathrm{e}}(\tau, \tau)$ is given. Then

$$
\begin{aligned}
u_{\mathrm{e}}(t)= & \int_{0}^{t} N_{\mathrm{e}}(t, \tau) C_{\mathrm{e}} x(\tau) \mathrm{d} \tau+\int_{0}^{t} N_{\mathrm{e}}(t, \tau) G_{\mathrm{e}} \mathrm{d} V_{\mathrm{e}}(\tau) \\
= & \int_{0}^{t} N_{\mathrm{e}}(t, \tau) C_{\mathrm{e}} \pi(t)(\tau) \mathrm{d} \tau+N_{\mathrm{e}}(t, t) \eta_{\mathrm{e}}(t)(t) \\
& -\int_{0}^{t}\left(\frac{\partial N_{\mathrm{e}}(t, \tau)}{\partial \tau}\right) \eta_{\mathrm{e}}(t)(\tau) \mathrm{d} \tau .
\end{aligned}
$$

Using the relation $\eta_{\mathrm{e}}(t)(t)=\eta_{\mathrm{e}}(t)\left(t_{f}\right)$ and defining

$$
I_{s, \tau}=\left\{\begin{array}{l}
0, s<\tau \\
I, s \geqslant \tau,
\end{array}\right.
$$

equation (1) can be rewritten as

$$
\begin{aligned}
& (\pi(t)(s), \phi)-(\pi(0)(s), \phi)=\int_{0}^{t}\left\langle A I_{s \tau} \pi(\tau)(s), \phi\right\rangle \mathrm{d} \tau+\int_{0}^{t}\left(B_{\mathrm{e}} I_{s \tau} \int_{0}^{t} N_{\mathrm{e}}(\tau, \sigma) C_{\mathrm{e}} \pi(\tau)(\sigma) \mathrm{d} \sigma, \phi\right) \mathrm{d} \tau \\
& \quad+\int_{0}^{t}\left(B_{\mathrm{e}} I_{s \tau}\left(N_{\mathrm{e}}(\tau, \tau) \eta_{\mathrm{e}}(\tau)\left(t_{f}\right)-\int_{0}^{\tau}\left(\frac{\partial N_{\mathrm{e}}(\tau, \sigma)}{\partial \sigma}\right) \eta_{\mathrm{e}}(\tau)(\sigma) \mathrm{d} \sigma\right), \phi\right) \mathrm{d} \tau \\
& \quad+\int_{0}^{t}\left(B_{\mathrm{p}} I_{s \tau} u_{\mathrm{p}}(\tau), \phi\right) \mathrm{d} \tau+\left(\int_{0}^{t} I_{s \tau} F \mathrm{~d} W(\tau), \phi\right), \quad \forall \phi \in V
\end{aligned}
$$

Furthermore, we clearly have

$$
\eta_{\mathrm{e}}(t)(s)=\int_{0}^{t} I_{s \tau} G_{\mathrm{e}} \mathrm{d} V_{\mathrm{e}}(\tau)
$$

and

$$
Y_{\mathrm{p}}(t)=\int_{0}^{t} C_{\mathrm{p}} \pi(\tau)\left(t_{f}\right) \mathrm{d} \tau+\int_{0}^{t} G_{\mathrm{p}} \mathrm{d} V_{\mathrm{p}}(\tau)
$$

Let $\mu$ be the Lebesgue-Stieltjes measure on $[0, T]$ which is the Lebesgue measure together with unit masses concentrated at $t=0$ and $t=T$. By $M^{2}(\bar{T} ; X)$ we shall mean the space of measurable functions from $[0, T]$ into a Hilbert space $X$ such that

$$
\int_{0}^{T}\|f\|_{\times}^{2} \mathrm{~d} \mu<\infty .
$$

This is a Hilbert space under the inner product

$$
[f, g]_{\times}=\left(f\left(t_{f}\right), g\left(t_{f}\right)\right)_{\times}+(f(0), g(0))_{\times}+\int_{0}^{t_{f}}(f(s), g(s))_{\times} \mathrm{d} s
$$

where $(\cdot, \cdot)_{\times}$denotes the inner product in $X$. On the other hand, let $\mu_{f}$ be the Lebesgue-Stieltjes measure on $[t, T]$, for fixed $t \in T$, which is the Lebesgue measure there with unit mass concentrated at $s=T$. We drop the suffix $X$ when $X=H$. By $M^{2}\left(\left(t, t_{f}\right] ; V\right)$ we shall mean the space of measurable functions $f$ from $[t, T]$ into $V$ such that

$$
\int_{1}^{T}|f|_{V}^{2} \mathrm{~d} \mu_{f}<\infty
$$

Now equation (8) can be represented as

$$
\begin{aligned}
& {[\pi(t), \phi]-[\pi(0), \phi]} \\
& \quad=\int_{0}^{t}\left[\left\langle A I_{\cdot \tau} \pi(t), \phi\right\rangle\right] \mathrm{d} \tau+\int_{0}^{t}\left[B_{\mathrm{e}} I_{\cdot \tau} \int_{0}^{\tau} N_{\mathrm{e}}(\tau, \sigma) C_{\mathrm{e}} \pi(\tau)(\sigma) \mathrm{d} \sigma, \phi\right] \mathrm{d} \tau
\end{aligned}
$$




$$
\begin{aligned}
& +\int_{0}^{t}\left[B_{\mathrm{e}} I_{\tau}\left(N_{\mathrm{e}}(\tau, \tau) \eta_{\mathrm{e}}(\tau)\left(t_{f}\right)-\int_{0}^{\tau}\left(\frac{\partial N_{\mathrm{e}}(\tau, \sigma)}{\partial \sigma}\right) \eta_{\mathrm{e}}(\tau)(\sigma) \mathrm{d} \sigma\right), \phi\right] \mathrm{d} \tau \\
& +\int_{0}^{t}\left[B_{\mathrm{p}} I_{\tau} u_{\mathrm{p}}(\tau), \phi\right] \mathrm{d} t+\left[\int_{0}^{t} I_{\tau} F \mathrm{~d} W(\tau), \phi\right] \forall \phi \in M^{2}(T ; V) \cap M^{2}(\bar{T} ; H)
\end{aligned}
$$

where

$$
\left[\left\langle A \phi_{1}, \phi_{2}\right\rangle\right] \triangleq\left\langle A \phi_{1}\left(t_{f}\right), \phi_{2}\left(t_{f}\right)\right\rangle+\left\langle A \phi_{1}(0), \phi_{2}(0)\right\rangle+\int_{0}^{t_{f}}\left\langle A \phi_{1}(s), \phi_{2}(s)\right\rangle \mathrm{d} s .
$$

Equation (9) can also be rewritten as

$$
\left[\eta_{\mathrm{e}}(t), \phi\right]_{R^{m_{\mathrm{e}}}}=\left[\int_{0}^{t} I_{{ }_{\tau}} G_{\mathrm{e}} \mathrm{d} V_{\mathrm{e}}(\tau), \phi\right]_{\mathbb{R}^{m_{\mathrm{e}}}} ; \quad \forall \phi \in M^{2}\left(\bar{T} ; \mathbb{R}^{m_{\mathrm{e}}}\right)
$$

\section{Theorem 3.1}

With the preceding notations,

$$
\eta_{\mathrm{e}} \in L^{2}\left(\Omega ; C\left(\bar{T} ; M^{2}\left(\bar{T} ; \mathbb{R}^{m_{\mathrm{e}}}\right)\right)\right)
$$

and

$$
\pi \in L^{2}\left(\Omega ; C\left(\bar{T} ; M^{2}(\bar{T} ; H)\right) \cap L^{2}\left(T ; M^{2}\left(\left(t, t_{f}\right] ; V\right)\right)\right) .
$$

Proof. From equation (13), it is easy to show that

$$
\left|\eta_{\mathrm{e}}(t)\right|_{M^{2}\left(\tilde{T}_{;} \mathbb{R}^{\left.m_{\mathrm{e}}\right)}\right.}^{2}=\left(1+t_{f}-t\right)\left|\int_{0}^{t} G_{\mathrm{e}} \mathrm{d} V_{\mathrm{e}}(\tau)\right|_{\mathbb{R}^{m_{\mathrm{e}}}}^{2}+\int_{0}^{1}\left|\int_{0}^{s} G_{\mathrm{c}} \mathrm{d} V_{\mathrm{c}}(\tau)\right|_{\mathbb{R}^{m_{\mathrm{e}}}}^{2} \mathrm{~d} s .
$$

Applying the Martingale inequality, (14) can be established.

On the other hand, we have

$$
\left[B_{\mathrm{e}} I_{\tau} \int_{0}^{\tau} N_{\mathrm{e}}(\tau, \sigma) C_{\mathrm{c}} \pi(\tau)(\sigma) \mathrm{d} \sigma, \pi(\tau)\right] \leqslant \text { const. }|\pi(\tau)|_{M^{2}(\bar{T} ; H)}^{2}
$$

and

$$
\begin{aligned}
& {\left[B_{\mathrm{e}} I_{\tau}\left(N_{\mathrm{e}}(\tau, \tau) \eta_{\mathrm{e}}(\tau)\left(t_{f}\right)-\int_{0}^{\tau}\left(\frac{\partial N_{\mathrm{e}}(\tau, \sigma)}{\partial \sigma}\right) \eta_{\mathrm{e}}(\tau)(\sigma) \mathrm{d} \sigma\right), \pi(\tau)\right]} \\
& \leqslant \text { const. }\left\{\left|\eta_{\mathrm{c}}(\tau)\right|_{M^{2}\left(\bar{T}_{:} \mathbb{R}^{m_{\mathrm{e}}}\right)}+|\pi(\tau)|_{M^{2}(\bar{T}: H)}^{2}+\int_{0}^{\tau}\left|\frac{\partial N_{\mathrm{e}}(\tau, \sigma)}{\partial \sigma}\right|_{L\left(\mathbb{R}^{\left.m_{\mathrm{e}}, H_{u}\right)}\right.}^{2} \mathrm{~d} \sigma\right\}
\end{aligned}
$$

Furthermore, from (A-1), it follows that

$$
\begin{aligned}
& -\left[\left\langle A I_{\cdot} \pi(\tau), \pi(\tau)\right\rangle\right]=-\left\langle A \pi(\tau)\left(t_{f}\right), \pi(\tau)\left(t_{f}\right)\right\rangle-\int_{\tau}^{t_{t}}\langle A \pi(\tau)(s), \pi(\tau)(s)\rangle \mathrm{d} s \\
& \geqslant \alpha_{1}\left|\pi(\tau)\left(t_{f}\right)\right|_{V}^{2}+\alpha_{1} \int_{\tau}^{t_{1}}|\pi(\tau)(s)|_{V}^{2} \mathrm{~d} s \geqslant \alpha_{1} \mid \pi(\tau)_{M^{2}\left(\tau \cdot t_{f} ; n\right)}^{2} .
\end{aligned}
$$

From the estimates (16), (17), (18) and the well-known results in stochastic differential equations (e.g. given in Bensoussan [5]), (15) follows.

Let us now express the criterion in terms of the new state variables. We have, in fact,

$$
\begin{aligned}
& J=\frac{1}{2} \int_{0}^{t_{t}}\left\{|\chi(t) \pi(t)|_{M^{2}\left(\bar{T}_{\mathrm{i}} H\right)}^{2}-|U(t) \pi(t)|_{H_{u}}^{2}-2\left(U(t) \pi(t), \gamma(t) \eta_{\mathrm{c}}(t)\right)_{H_{u}}\right. \\
&\left.-\left|\gamma(t) \eta_{\mathrm{c}}(t)\right|_{H_{u}}^{2}\right\} \mathrm{d} t+\frac{1}{2} \int_{0}^{t_{u}^{\prime}}\left|R_{\mathrm{r}} u_{\mathrm{p}}(t)\right|_{H_{u}}^{2} \mathrm{~d} t
\end{aligned}
$$


where

$$
\begin{gathered}
\chi(t)=I_{s t}, Q \\
U(t) \pi(t)=R_{\mathrm{e}} \int_{0}^{t} N_{\mathrm{e}}(t, \tau) C_{\mathrm{e}} \pi(t)(\tau) \mathrm{d} t
\end{gathered}
$$

and

$$
\gamma(t) \eta_{\mathrm{c}}(t)=R_{\mathrm{e}}\left(N_{\mathrm{e}}(t, t) \eta_{\mathrm{e}}(t)\left(t_{f}\right)-\int_{0}^{t}\left(\frac{\partial N_{\mathrm{c}}(t, \tau)}{\partial t}\right) \eta_{\mathrm{c}}(t)(\tau) \mathrm{d} \tau\right)
$$

The pursuer wants to choose $u_{\mathrm{p}}(\mathrm{t})$ so as to minimize the expected value of (19) subject to (11) and (13).

\section{SOLUTION OF THE CONTROL PROBLEM}

In this section, we assume that

$$
\chi^{*}(t) \chi(t)-U^{*}(t) U(t) \text { is nonnegative definite. }
$$

Under the preceding assumption, by using the stochastic maximum principle, we can obtain the necessary and sufficient condition for optimality of $u_{\mathrm{p}}^{0}$.

\section{Theorem 4.1}

The optimal control $u_{\mathrm{p}}^{0}(t)$ is characterized by the following variational inequality

$$
\begin{gathered}
\int_{0}^{t_{t}}\left\{\left[B_{\mathrm{p}} I_{t}\left(\tilde{u}_{\mathrm{p}}(t)-u_{\mathrm{p}}^{0}(t)\right), E\left[q_{\mathrm{ad}}(t) \mid \mathscr{Y}_{t}^{\mathrm{p}}\right]\right]-\left(R_{\mathrm{p}}\left(\tilde{u}_{\mathrm{p}}(t)-u_{\mathrm{p}}^{0}(t)\right), R_{\mathrm{p}} u_{\mathrm{p}}^{0}(t)\right)_{H_{u}}\right\} \mathrm{d} t \geqslant 0 \text { a.s. } \\
\forall \tilde{u}_{\mathrm{p}} \in \text { class of admissible controls, }
\end{gathered}
$$

where $\mathscr{Y}_{t}^{\mathrm{p}}=\sigma\left\{Y_{\mathrm{p}}(s) ; 0 \leqslant s \leqslant t\right\}$ and $q_{\mathrm{ad}}(t)$ is the solution of an adjoint system

$$
\begin{gathered}
-\left[\frac{\mathrm{d} q_{\mathrm{ad}}(t)}{\mathrm{d} t}, \phi(t)\right]=\left[\left\langle A I_{t} \phi(t), q_{\mathrm{ad}}(t)\right\rangle\right]+\left[B_{\mathrm{e}} I_{t} \int_{0}^{t} N_{\mathrm{e}}(t, \sigma) C_{\mathrm{e}} \phi(t)(\sigma) \mathrm{d} \sigma, q_{\mathrm{ad}}(t)\right] \\
+[\chi(t) \phi(t), \chi(t) \pi(t)]-(U(t) \phi(t), U(t) \pi(t))_{H_{u}}-2\left(U(t) \phi(t), \gamma(t) \eta_{\mathrm{e}}(t)\right)_{H_{u}} \\
{\left[q_{\mathrm{ad}}\left(t_{f}\right), \phi\left(t_{f}\right)\right]=0 \quad \forall \phi \in C\left(\bar{T} ; M^{2}(\bar{T} ; H)\right) \cap L^{2}\left(\bar{T} ; M^{2}\left(\left(t, t_{f}\right] ; V\right)\right) .}
\end{gathered}
$$

Proof. From (19), we have the following necessary condition for optimality:

$$
\begin{aligned}
E \int_{0}^{t_{f}}\left\{[\chi(t) z(t), \chi(t) \pi(t)]-(U(t) z(t), U(t) \pi(t))_{H_{u}}\right. \\
\left.\quad-2\left(U(t) z(t), \gamma(t) \eta_{\mathrm{c}}(t)\right)_{H_{u}}\right\} \mathrm{d} t+E \int_{0}^{t_{f}}\left\{R_{\mathrm{p}}\left(\tilde{u}_{\mathrm{p}}(t)-u_{\mathrm{p}}^{0}(t), R_{\mathrm{p}} u_{\mathrm{p}}^{0}(t)\right)_{H_{u}}\right\} \mathrm{d} t \geqslant 0 \\
\quad \forall \tilde{u}_{\mathrm{p}}, u_{\mathrm{p}}^{0} \in \text { class of admissible controls } \dagger
\end{aligned}
$$

where $z(t)$ is the homogeneous solution of (11); i.e.

$$
\begin{gathered}
{\left[\frac{\mathrm{d} z(t)}{\mathrm{d} t}, \phi(t)\right]=\left[\left\langle A I_{,} z(t), \phi(t)\right\rangle\right]+\left[B_{\mathrm{e}} I_{\cdot t} \int_{0}^{t} N_{\mathrm{e}}(t, \sigma) C_{\mathrm{e}} z(\sigma) \mathrm{d} \sigma, \phi(t)\right]} \\
+\left[B_{\mathrm{p}} I_{t}\left(\tilde{u}_{\mathrm{p}}(t)-u_{\mathrm{p}}^{0}(t)\right), \phi(t)\right] \\
{[z(0), \phi(0)]=0 \quad \forall \phi \in C\left(\bar{T} ; M^{2}(\bar{T} ; H)\right) \cap L^{2}\left(\bar{T} ; M^{2}\left(\left(t, t_{f}\right]\right) ; V\right) .}
\end{gathered}
$$


Calculating $\left[z(t), q_{\mathrm{ad}}(t)\right]$ from (22) and (24), equation (23) implies that

$$
\left.E \int_{0}^{t_{f}}\left\{B_{\mathrm{p}} I_{,}\left(\tilde{u}_{\mathrm{p}}(t)-u_{\mathrm{p}}^{0}(t)\right), q_{\mathrm{ad}}(t)\right]-\left(R_{\mathrm{p}}\left(\tilde{u}_{\mathrm{p}}(t)-u_{\mathrm{p}}^{0}(t)\right), R_{\mathrm{p}} u_{\mathrm{p}}^{0}(t)\right)_{H_{u}}\right\} \mathrm{d} t \geqslant 0 .
$$

Equation (21) can now be easily derived using the properties of conditional expectations.

\section{Theorem 4.2}

The optimal control $u_{\mathrm{p}}^{0}(t)$ is determined by

$$
u_{\mathrm{p}}^{0}(t)=-\left(R_{\mathrm{p}}^{*} R_{\mathrm{p}}\right)^{-1} B_{\mathrm{p}}^{*}\left\{E\left[q_{\mathrm{ad}}(t)\left(t_{f}\right) \mid \mathscr{Y}_{\mathrm{t}}^{\mathrm{p}}\right]+\int_{t}^{t_{t}} E\left[\left.q_{\mathrm{ad}}(t)(s)\right|^{\mathrm{p}}\right] \mathrm{d} s\right\} .
$$

Proof. From the definition of inner product in $M^{2}(T ; X)$,

$$
\begin{aligned}
\int_{0}^{t_{f}} & {\left[B_{\mathrm{p}} I_{t}\left(\tilde{u}_{\mathrm{p}}(t)-u_{\mathrm{p}}^{0}(t)\right), E\left[q_{\mathrm{ad}}(t) \mid \mathscr{Y}_{t}^{\mathrm{p}}\right]\right] \mathrm{d} t=\int_{0}^{t_{f}}\left\{\left(B_{\mathrm{p}}\left(\tilde{u}_{\mathrm{p}}(t)-u_{\mathrm{p}}^{0}(t)\right), E\left[q_{\mathrm{ad}}(t)\left(t_{f}\right) \mid \mathscr{Y}_{t}^{\mathrm{P}}\right]\right)\right.} \\
& \left.+\int_{0}^{t_{f}}\left(B_{\mathrm{p}} I_{s t}\left(\tilde{u}_{\mathrm{p}}(t)-u_{\mathrm{p}}^{0}(t)\right), E\left[q_{\mathrm{ad}}(t)(s) \mid \mathscr{Y}_{t}^{\mathrm{p}}\right]\right) \mathrm{d} s\right\} \mathrm{d} t \\
& =\int_{0}^{t f}\left(\tilde{u}_{\mathrm{p}}(t)-u_{\mathrm{p}}^{0}(t), B_{\mathrm{p}}^{*}\left(E\left[q_{\mathrm{ad}}(t)\left(t_{f}\right) \mid \mathscr{Y}_{t}^{\mathrm{p}}\right]+\int_{t}^{t_{f}} E\left[q_{\mathrm{ad}}(t)(s) \mid \mathscr{Y}_{t}^{\mathrm{p}}\right] \mathrm{d} s\right)\right)_{H_{u}} \mathrm{~d} t .
\end{aligned}
$$

From (21) and (27), we can readily derive (26).

\section{Theorem 4.3}

We have

$\left[E\left[q_{\mathrm{ad}}(t) \mid \mathscr{Y}_{t}^{\mathrm{p}}\right], \phi(t)\right]=\left[\mathscr{K}_{1}(t) E\left[\pi(t) \mid \mathscr{Y}_{t}^{\mathrm{p}}\right], \phi(t)\right]+\left[\mathscr{K}_{2}(t) E\left[\eta_{\mathrm{e}}(t) \mid \mathscr{Y}_{l}^{\mathrm{p}}\right], \phi(t)\right]$

$$
\forall \phi \in C\left(\bar{T} ; M^{2}(\bar{T} ; H)\right) \cap L^{2}\left(\bar{T} ; M^{2}\left(\left(t, t_{f}\right] ; V\right)\right)
$$

where

$$
\begin{aligned}
& -\left[\frac{\mathrm{d} \mathscr{K}_{1}(t)}{\mathrm{d} t} \phi_{1}, \phi_{2}\right]=\left[\left\langle A I_{,}, \phi_{1}, \mathscr{K}_{1}^{*}(t) \phi_{2}\right\rangle\right]+\left[\left\langle A I_{\cdot}, \phi_{2}, \mathscr{K}_{1}(t) \phi_{1}\right\rangle\right] \\
& +\left[B_{\mathrm{e}} I_{1} \int_{0}^{t} N_{\mathrm{c}}(t, \sigma) C_{\mathrm{e}} \phi_{1}(t)(\sigma) \mathrm{d} \sigma, \mathscr{K}_{1}^{*}(t) \phi_{2}\right]+\left[B_{\mathrm{c}} I_{1} \int_{0}^{t} N_{\mathrm{c}}(t, \sigma) C_{\mathrm{e}} \phi_{2}(t)(\sigma) \mathrm{d} \sigma, \mathscr{K}_{1}(t) \phi_{1}\right] \\
& -\left[B_{\mathrm{p}} I_{t}\left(R_{\mathrm{p}}^{*} R_{\mathrm{p}}\right)^{-1} B_{\mathrm{p}}^{*}\left\{\mathscr{K}_{1}(t)\left(t_{f}\right) \phi_{1}+\int_{1}^{t_{f}} \mathscr{K}_{1}(t)(s) \phi_{1} \mathrm{~d} s\right\}, \mathscr{K}_{1}^{*}(t) \phi_{2}\right] \\
& +\left[\chi \phi_{1}, \chi \phi_{2}\right]-\left(U \phi_{1}, U \phi_{2}\right)_{H_{u}} \\
& {\left[\mathscr{K}_{1}\left(t_{f}\right) \phi_{1}, \phi_{2}\right]=0 \quad \forall \phi_{1}, \phi_{2} \in C\left(\bar{T} ; M^{2}(\bar{T} ; H)\right) \cap L^{2}\left(\bar{T} ; M^{2}\left(\left(t, t_{f}\right] ; V\right)\right)}
\end{aligned}
$$

and

$$
\begin{gathered}
-\left[\frac{\mathrm{d} \mathscr{K}_{2}(t)}{\mathrm{d} t} \phi_{1}, \phi_{2}\right]=\left[\left\langle A I_{t} \phi_{2}, \mathscr{K}_{2}(t) \phi_{1}\right\rangle\right]+\left[B_{\mathrm{e}} I_{\cdot} \int_{0}^{t} N_{\mathrm{e}}(t, \sigma) C_{\mathrm{e}} \phi_{2}(t)(\sigma) \mathrm{d} \sigma, \mathscr{K}_{2}(t) \phi_{1}\right] \\
-\left[B_{\mathrm{p}} I_{t}\left(R_{\mathrm{p}}^{*} R_{\mathrm{p}}\right)^{-1} B_{\mathrm{p}}^{*}\left\{\mathscr{K}_{2}(t)\left(t_{f}\right) \phi_{1}+\int_{t}^{t_{t}} \mathscr{K}_{2}(t)(s) \phi_{1} \mathrm{~d} s\right\}, \mathscr{K}_{1}^{*}(t) \phi_{2}\right] \\
+\left[B_{\mathrm{e}} I_{t}\left(N_{\mathrm{c}}(t, t) \phi_{1}(t)\left(t_{f}\right)-\int_{0}^{t}\left(\frac{\partial N_{\mathrm{e}}(t, \sigma)}{\partial \sigma}\right) \phi_{1}(t)(\sigma) \mathrm{d} \sigma\right), \mathscr{K}_{1}^{*}(\tau) \phi_{2}\right]-2\left(U \phi_{2}, \gamma \phi_{1}\right)_{H_{u}} \\
{\left[\mathscr{K}_{2}\left(\tau_{f}\right) \phi_{1}, \phi_{2}\right]=0 \quad \forall \phi_{1}, \phi_{2} \in C\left(\bar{T} ; M^{2}\left(\bar{T} ; \mathbb{R}^{m_{\mathrm{e}}}\right)\right)}
\end{gathered}
$$


Proof. We assume that the observation noise $V_{\mathrm{p}}$ is independent of $W$ and $\pi(0)$. Define

$$
\hat{\pi}_{t}(\tau)=E\left[\pi(\tau) \mid \mathscr{Y}_{t}^{\mathrm{p}}\right], \quad \hat{\eta}_{\mathrm{el}}(\tau)=E\left[\left.\eta_{\mathrm{c}}(\tau)\right|_{y_{t}^{\mathrm{p}}} ^{\mathrm{p}}\right], \quad \tau \geqslant t
$$

and

$$
\hat{q}_{\mathrm{adr}}(\tau)=E\left[q_{\mathrm{ad}}(\tau) \mid \mathscr{Y}_{\mathrm{t}}^{\mathrm{p}}\right], \quad \tau \geqslant t
$$

From equations (22), (11) and (13), we get

$$
\begin{aligned}
& \left\{\begin{aligned}
-\left[\frac{\mathrm{d} \hat{q}_{\mathrm{ad} t}(\tau)}{\mathrm{d} \tau}, \phi\right]= & {\left[\left\langle A I_{\cdot \tau} \phi, \hat{q}_{\mathrm{ad} t}(\tau)\right\rangle\right]+\left[B_{\mathrm{e}} I_{\cdot \tau} \int_{0}^{\tau} N_{\mathrm{e}}(\tau, \sigma) C_{\mathrm{e}} \phi(\tau)(\sigma) \mathrm{d} \sigma, \hat{q}_{\mathrm{ad} t}(\tau)\right] } \\
& +\left[\chi \phi, \chi \hat{\pi}_{t}(\tau)\right]-\left(U \phi, U \hat{\pi}_{t}(\tau)\right)_{H_{u}}-2\left(U \phi, \gamma \hat{\eta}_{\mathrm{et}}(\tau)\right)_{H_{u}}
\end{aligned}\right. \\
& {\left[\hat{q}_{\mathrm{ad} t}\left(t_{f}\right), \phi\right]=0} \\
& {\left[\frac{\mathrm{d} \hat{\pi}_{t}(\tau)}{\mathrm{d} \tau}, \phi\right]=\left[\left\langle A I_{\cdot} \hat{\pi}_{t}(\tau), \phi\right\rangle\right]} \\
& +\left[B_{\mathrm{c}} I_{\cdot \tau} \int_{0}^{\tau} N_{\mathrm{e}}(\tau, \sigma) C_{\mathrm{e}} \hat{\pi}_{t}(\tau)(\sigma) \mathrm{d} \sigma, \phi\right] \\
& +\left[B_{\mathrm{e}} I_{\tau}\left(N_{\mathrm{c}}(\tau, \tau) \hat{\eta}_{\mathrm{et}}(\tau)\left(t_{f}\right)-\int_{0}^{\tau}\left(\frac{\partial N_{\mathrm{c}}(\tau, \sigma)}{\partial \sigma}\right) \hat{\eta}_{\mathrm{et}}(\tau)(\sigma) \mathrm{d} \sigma\right), \phi\right] \\
& -\left[B_{\mathrm{p}} I_{\cdot \tau}\left(R_{\mathrm{p}}^{*} R_{\mathrm{p}}\right)^{-1} B_{\mathrm{p}}^{*}\left\{\hat{q}_{\mathrm{ad}} t(\tau)\left(t_{f}\right)+\int_{\tau}^{t_{\mathrm{t}}} \hat{q}_{\mathrm{ad} t}(\tau)(s) \mathrm{d} s\right\}, \phi\right] \\
& {\left[\hat{\pi}_{t}(t), \phi\right]=\left[E\left[\pi(t) \mid \mathscr{Y}_{t}^{\mathrm{p}}\right], \phi\right]}
\end{aligned}
$$

and

$$
\left\{\begin{aligned}
{\left[\frac{\mathrm{d} \hat{\eta}_{\mathrm{et}}(\tau)}{\mathrm{d} \tau}, \phi\right]_{\mathbb{R}^{m_{\mathrm{e}}}} } & =0 \\
{\left[\hat{\eta}_{\mathrm{et}}(t), \phi\right]_{\mathbb{R}_{\mathrm{e}}} } & =\left[E\left[\eta_{\mathrm{e}}(t) \mid \mathscr{Y}_{\mathrm{i}}^{\mathrm{p}}\right], \phi\right]_{\mathbb{R}^{m_{\mathrm{e}}}} \quad \forall \phi \in C\left(T ; M^{2}\left(T ; \mathbb{R}^{m_{\mathrm{e}}}\right)\right) .
\end{aligned}\right.
$$

Applying the decoupling method as given in Bensoussan and Viot [7], we have

$$
\hat{q}_{\mathrm{ad} t}(\tau)=\mathscr{K}_{1}(t) \hat{\pi}_{t}(\tau)+\mathscr{K}_{2}(t) \hat{\eta}_{\mathrm{er}}(\tau),
$$

where $\mathscr{K}_{1}$ and $\mathscr{K}_{2}$ are solutions of (29) and (30), respectively.

\section{Theorem 4.4}

Define

$$
\hat{\pi}(t)=E\left[\pi(t) \mid \mathscr{Y}_{t}^{\mathrm{p}}\right] \quad \text { and } \quad \hat{\eta}_{\mathrm{c}}(t)=E\left[\eta_{\mathrm{c}}(t) \mid \mathscr{Y}_{t}^{\mathrm{p}}\right] .
$$

They are characterized by the following linear estimators:

$$
\begin{aligned}
{[\hat{\pi}(t), \phi]=} & \int_{0}^{t}\left\{\left[\left\langle A I_{\tau} \hat{\pi}(\tau), \phi\right\rangle\right]+\left[B_{\mathrm{e}} I_{\tau} \int_{0}^{\tau} N_{\mathrm{e}}(\tau, \sigma) C_{\mathrm{e}} \hat{\pi}(\tau)(\sigma) \mathrm{d} \sigma, \phi\right]\right. \\
& +\left[B_{\mathrm{e}} I_{\tau}\left(N_{\mathrm{e}}(\tau, \tau) \hat{\eta}_{\mathrm{e}}(\tau)\left(t_{f}\right)-\int_{0}^{\tau}\left(\frac{\partial N_{\mathrm{e}}(\tau, \sigma)}{\partial \sigma}\right) \hat{\eta}_{\mathrm{e}}(\tau)(\sigma) \mathrm{d} \sigma\right), \phi\right] \\
& -\left[B _ { \mathrm { p } } I _ { \tau } ( R _ { \mathrm { p } } ^ { * } R _ { \mathrm { p } } ) ^ { - 1 } B _ { \mathrm { p } } ^ { * } \left\{\mathscr{K}_{1}(\tau)\left(t_{f}\right) \hat{\pi}(\tau)+\mathscr{K}_{2}(\tau)\left(t_{f}\right) \hat{\eta}_{\mathrm{e}}(\tau)\right.\right.
\end{aligned}
$$




$$
\begin{aligned}
& \left.\left.\left.+\int_{\tau}^{t_{f}}\left(\mathscr{K}_{1}(\tau)(s) \hat{\pi}(\tau)+\mathscr{K}_{2}(\tau)(s) \hat{\eta}_{\mathrm{e}}(\tau)\right) \mathrm{d} s\right\}, \phi\right]\right\} \mathrm{d} \tau \\
& +\int_{0}^{t}\left[\mathscr{P}_{1}(\tau) C_{\mathrm{p}}^{*}\left(G_{\mathrm{p}} \mathscr{\mathscr { p }}_{\mathrm{p}} G_{\mathrm{p}}^{*}\right)^{-1}\left(\mathrm{~d} Y_{\mathrm{p}}(\tau)-C_{\mathrm{p}} \hat{\pi}(\tau) \mathrm{d} \tau\right), \phi\right] \\
& \forall \phi \in C\left(\bar{T} ; M^{2}(\bar{T} ; H)\right) \cap L^{2}\left(\bar{T} ; M^{2}\left(\left(t, t_{f}\right] ; V\right)\right)
\end{aligned}
$$

and

$$
\begin{aligned}
{\left[\hat{\eta}_{\mathrm{e}}(t), \phi\right]_{\mathbb{R}_{\mathrm{e}}}=} & \int_{0}^{t}\left[\mathscr{P}_{2}(\tau) C_{\mathrm{p}}^{*}\left(G_{\mathrm{p}} \mathscr{V}_{\mathrm{p}} G_{\mathrm{p}}^{*}\right)^{-1}\left(\mathrm{~d} Y_{\mathrm{p}}(\tau)-C_{\mathrm{p}} \hat{\pi}(\tau) \mathrm{d} \tau\right), \phi\right]_{\mathbb{R}^{m_{\mathrm{e}}}} \\
& \forall \phi \in C\left(\bar{T} ; M^{2}\left(\bar{T} ; \mathbb{R}^{m_{\mathrm{e}}}\right)\right)
\end{aligned}
$$

where $\mathscr{P}_{1}(t)$ and $\mathscr{P}_{2}(t)$ are defined by

$$
\left[\phi_{1}, \mathscr{P}_{1}(t) \phi_{2}\right]=E\left\{\left[\phi_{1}, \pi(t)\right]\left[\pi(t), \phi_{2}\right]\right\}-E\left\{\left[\phi_{1}, \hat{\pi}(t)\right]\left[\hat{\pi}(t), \phi_{2}\right]\right\}
$$

and

$\left[\phi, \mathscr{P}_{2}(t) \phi_{1}\right]_{\mathbb{R}^{m_{\mathrm{e}}}}=E\left\{\left[\phi, \eta_{\mathrm{e}}(t)\right]_{\mathbb{R}^{m_{\mathrm{e}}}}\left[\pi(t), \phi_{1}\right]\right\}-E\left\{\left[\phi, \hat{\eta}_{\mathrm{e}}(t)\right]_{\mathbb{R}^{\prime} m_{\mathrm{e}}}\left[\hat{\pi}(t), \phi_{1}\right]\right\}$

$$
\forall \phi_{1}, \phi_{2} \in C\left(\bar{T} ; M^{2}(\bar{T} ; H)\right) \cap L^{2}\left(\bar{T} ; M^{2}\left(\left(t, t_{f}\right] ; V\right)\right)
$$

and

$$
\forall \phi \in C\left(\bar{T} ; M^{2}\left(\bar{T} ; \mathbb{R}^{m_{\mathrm{e}}}\right)\right) .
$$

Proof. Applying the linear filtering theory in Hilbert spaces, above results are easily derived.

\section{Theorem 4.5}

$\mathscr{P}_{1}$ and $\mathscr{P}_{2}$ are solutions of the following Riccati equations:

$$
\begin{aligned}
& {\left[\frac{\mathrm{d} \mathscr{P}_{1}(t)}{\mathrm{d} t} \phi_{1}, \phi_{2}\right]=\left[\left\langle A I_{1} \mathscr{P}_{1}(t) \phi_{1}, \phi_{2}\right\rangle\right]+\left[\left\langle A I_{1}, \phi_{1}, \mathscr{P}_{1}(t) \phi_{2}\right\rangle\right]} \\
& +\left[B_{\mathrm{c}} I_{1} \int_{0}^{t} N_{\mathrm{c}}(t, \sigma) C_{\mathrm{c}} \mathscr{P}_{1}(t)(\sigma) \phi_{1} \mathrm{~d} \sigma, \phi_{2}\right]+\left[B_{\mathrm{c}} I_{t} \int_{0}^{t} N_{\mathrm{c}}(t, \sigma) C_{\mathrm{c}} \mathscr{P}_{1}(t)(\sigma) \phi_{2} \mathrm{~d} \sigma, \phi_{1}\right] \\
& +\left[B_{\mathrm{c}} I_{t}\left(N_{\mathrm{c}}(t, t) \mathscr{P}_{2}(t)\left(t_{f}\right) \phi_{1}-\int_{0}^{t} \frac{\partial N_{\mathrm{c}}(t, \sigma)}{\partial \sigma} \mathscr{P}_{2}(t)(\sigma) \phi_{1} \mathrm{~d} \sigma\right), \phi_{2}\right] \\
& +\left[B_{\mathrm{c}} I_{1}\left(N_{\mathrm{c}}(t, t) \mathscr{P}_{2}(t)\left(t_{f}\right) \phi_{2}-\int_{0}^{t} \frac{\partial N_{\mathrm{c}}(t, \sigma)}{\partial \sigma} \mathscr{P}_{2}(t)(\sigma) \phi_{2} \mathrm{~d} \sigma\right), \phi_{1}\right] \\
& -\left[\mathscr{P}_{1}(t) C_{\mathrm{p}}^{*}\left(G_{\mathrm{p}} \mathscr{Y}_{\mathrm{p}} G_{\mathrm{p}}^{*}\right){ }^{\prime} C_{\mathrm{p}} \mathscr{P}_{1}(t) \phi_{1}, \phi_{2}\right] \\
& +\left[I_{t} F \mathscr{W} F^{*}\left(\phi_{1}(t)\left(t_{f}\right)+\int_{t}^{t_{1}} \phi_{1}(t)(s) \mathrm{d} s\right), \phi_{2}\right]
\end{aligned}
$$

$\left[\mathscr{P}_{1}(0) \phi_{1}, \phi_{2}\right]=\left(P_{0}\left(\phi_{1}(t)\left(t_{f}\right)+\phi_{1}(t)(0)+\int_{0}^{t_{1}} \phi_{1}(t)(s) \mathrm{d} s\right), \phi_{2}(t)\left(t_{f}\right)\right.$

$$
\left.+\phi_{2}(t)(0)+\int_{0}^{t} \phi_{2}(t)(s) \mathrm{d} s\right)
$$

$\forall \phi_{1}, \phi_{2} \in C\left(\bar{T} ; M^{2}(\bar{T} ; H)\right) \cap L^{2}\left(\bar{T} ; M^{2}\left(\left(0, t_{f}\right] V\right)\right)$ 
and

$$
\begin{gathered}
{\left[\frac{\mathrm{d} \mathscr{P}_{2}(t)}{\mathrm{d} t} \phi_{1}, \phi\right]_{\mathbb{R}_{\mathrm{e}}}=-\left[\mathscr{P}_{2}(t) C_{\mathrm{p}}^{*}\left(G_{\mathrm{p}} \mathscr{V}_{\mathrm{p}} G_{\mathrm{p}}^{*}\right)^{-1} C_{\mathrm{p}} \mathscr{P}_{1}(t) \phi_{1}, \phi\right]_{\mathbb{R}^{m_{\mathrm{e}}}}} \\
+\left[\left\langle A I_{,}, \phi_{1}, \mathscr{P}_{3}(t) \phi\right\rangle\right]+\left[B_{\mathrm{e}} I_{t} \int_{0}^{t} N_{\mathrm{c}}(t, \tau) C_{\mathrm{e}} \phi_{1}(\tau)(\sigma) \mathrm{d} \sigma, \mathscr{P}_{3}(t) \phi\right] \\
+\left[B_{\mathrm{e}} I_{t}\left(N_{\mathrm{e}}(t, t) \mathscr{P}_{4}(t)\left(t_{f}\right) \phi-\int_{0}^{t} \frac{\partial N_{\mathrm{c}}(\tau, \sigma)}{\partial \sigma} \mathscr{P}_{4}(t)(\sigma) \phi \mathrm{d} \sigma\right), \phi_{1}\right] \\
\mathscr{P}_{2}(0)=0 \forall \phi \in C\left(\bar{T} ; M^{2}\left(\bar{T} ; \mathbb{R}^{m_{\mathrm{e}}}\right)\right)
\end{gathered}
$$

where $\mathscr{P}_{3}(t)$ is defined by

$$
\left[\mathscr{P}_{3}(t) \phi, \phi_{1}\right]=\left[\phi, \mathscr{P}_{2}(t) \phi_{1}\right]_{\mathbb{R}^{m_{\mathrm{e}}}}
$$

and $\mathscr{P}_{4}$ is a solution of

$$
\begin{gathered}
{\left[\frac{\mathrm{d} \mathscr{P}_{4}(t)}{\mathrm{d} t} \phi_{1}, \phi_{2}\right]_{\mathbb{R}_{\mathrm{e}}}=\left[I, G_{\mathrm{e}} \mathscr{Y}_{\mathrm{c}} G_{\mathrm{c}}^{*}\left(\phi_{1}(t)\left(t_{f}\right)+\int_{1}^{t_{t}} \phi_{1}(t)(s) \mathrm{d} s\right), \phi_{2}\right]_{\mathbb{R}^{m_{\mathrm{e}}}}} \\
-\left[\mathscr{P}_{2}(t) C_{\mathrm{p}}^{*}\left(G_{\mathrm{p}} \mathscr{Y}_{\mathrm{p}} G_{\mathrm{p}}^{*}\right)^{-1} C_{\mathrm{p}} \mathscr{P}_{3}(t) \phi_{1}, \phi_{2}\right]_{\mathbb{R}^{m_{\mathrm{c}}}} \\
\mathscr{P}_{4}(0)=0 \quad \forall \phi_{1}, \phi_{2} \in C\left(\bar{T} ; M^{2}\left(\bar{T} ; \mathbb{R}^{m_{\mathrm{c}}}\right)\right) .
\end{gathered}
$$

Proof. Here the last term of the r.h.s. of (37a) is derived, because the remaining parts are easy consequences of the filtering theory. From the definition of inner product in $M^{2}(\bar{T} ; X)$, it follows that

$$
\begin{aligned}
E\left\{\int_{0}^{t}\left[I_{\tau} F \mathrm{~d} W(\tau), \phi_{1}\right] \int_{0}^{t}\left[I_{\tau} F \mathrm{~d} W(\tau), \phi_{2}\right]\right\}=E\left\{\int _ { 0 } ^ { t } \left(\phi_{1}(t)\left(t_{f}\right)\right.\right. \\
\left.\left.\quad+\int_{\tau}^{t} \phi_{1}(t)(s) \mathrm{d} s, F \mathrm{~d} W(\tau)\right) \times \int_{0}^{t}\left(\phi_{2}(t)\left(t_{f}\right)+\int_{\tau}^{t_{f}} \phi_{2}(t)(s) \mathrm{d} s, F \mathrm{~d} W(\tau)\right)\right\} \\
=\int_{0}^{t}\left(\phi_{1}(t)\left(t_{f}\right)+\int_{\tau}^{t_{t}} \phi_{1}(t)(s) \mathrm{d} s, F \mathscr{W} F^{*}\left(\phi_{2}(t)\left(t_{f}\right)+\int_{\tau}^{t_{f}} \phi_{2}(t)(s) \mathrm{d} s\right)\right) \mathrm{d} \tau \\
=\left[I_{t} F \mathscr{W} F^{*}\left(\phi_{1}(t)\left(t_{f}\right)+\int_{t}^{t_{f}} \phi_{1}(t)(s) \mathrm{d} s\right), \phi_{2}\right] .
\end{aligned}
$$

Now defining

$$
z(t)=\left(\begin{array}{c}
\hat{\pi}(t) \\
\hat{\eta}_{\mathrm{e}}(t)
\end{array}\right)
$$

state estimate equations (34) and (35) are represented by

$$
z(t)=\int_{0}^{t} \mathscr{A}(\tau) z(\tau) \mathrm{d} \tau+\int_{0}^{t}\left(\begin{array}{cc}
\mathscr{P}_{1}(\tau) & 0 \\
0 & \mathscr{P}_{2}(t)
\end{array}\right) C_{\mathrm{p}}^{*}\left(G_{\mathrm{p}} \mathscr{V}_{\mathrm{p}} G_{\mathrm{p}}^{*}\right)^{-1} \mathrm{~d} Y(\tau)
$$

where the operator $\mathscr{A}(t)$ belongs to

$$
\angle\left(M^{2}\left(\left(t, t_{f}\right] ; V\right) \times M^{2}\left(T ; \mathbb{R}^{m_{\mathrm{e}}}\right) ; \quad M^{2}\left(\left(t, t_{f}\right] ; V^{\prime}\right) \times M^{2}\left(\bar{T} ; \mathbb{R}^{m_{\mathrm{e}}}\right)\right),
$$

and then there exists a fundamental solution $\Psi(t, \sigma)$ of the equation $\mathrm{d} z / \mathrm{d} t+\mathscr{A}(t) z=0$ in $V^{\prime} \times \mathbb{R}^{m_{\mathrm{e}}}$. Consequently, from (10), (17) and (41), the optimal control $u_{\mathrm{p}}^{0}(t)$ becomes

$$
\begin{aligned}
u_{\mathrm{p}}^{0}(t)= & -\int_{0}^{t}\left(R_{\mathrm{p}}^{*} R_{\mathrm{p}}\right)^{-1} B_{\mathrm{p}}^{*}\left\{\left(\begin{array}{cc}
\mathscr{K}_{1}(t)\left(t_{f}\right) & 0 \\
0 & \mathscr{K}_{2}(t)\left(t_{f}\right)
\end{array}\right) \Psi(t, \tau)\left(\begin{array}{cc}
\mathscr{P}_{2}(\tau) & 0 \\
0 & \mathscr{P}_{2}(\tau)
\end{array}\right)\right. \\
& \left.+\int_{1}^{t_{j}}\left(\begin{array}{cc}
\mathscr{K}_{1}(t)(s) & 0 \\
0 & \mathscr{K}_{2}(t)(s)
\end{array}\right) \Psi(t, \tau)\left(\begin{array}{cc}
\mathscr{P}_{1}(\tau) & 0 \\
0 & \mathscr{P}_{2}(\tau)
\end{array}\right) \mathrm{d} s\right\} C_{\mathrm{p}}^{*}\left(G_{\mathrm{p}} \mathscr{V}_{\mathrm{p}} G_{\mathrm{p}}^{*}\right)^{-1} \mathrm{~d} Y_{\mathrm{p}}(\tau) .
\end{aligned}
$$


After tedious calculations, it can be checked that $N_{\mathrm{n}}(t, \tau)$ satisfies all the conditions stated in Section 1, under the condition for $N_{\mathrm{c}}(t, \tau)$-gain which satisfies same conditions stated in Section 1.

The right-hand side of (42) obviously depends on $N_{\mathrm{c}}$ and thus (42) gives the first relation connecting $N_{\mathrm{F}}$ and $N_{\mathrm{c}}$. Interchanging the roles of the players, another relation between $N_{\mathrm{p}}$ and $N_{\mathrm{c}}$ is obtained. It should be noted that this relation is derived from the necessary condition for optimality

\section{REPRESENTATION OF THE GAIN OPERATORS}

The control and filter gain operators $\mathscr{K}_{i}(t)$ and $\mathscr{P}_{i}(t)$ have integral kernels. In this section, we explicitly derive equations satisfied by the kernels corresponding to the operator $\mathscr{K}_{1}(t)$. Schwartz's kernel theorem implies that $\mathscr{K}_{1}(t)$ is represented by

$$
\begin{aligned}
\mathscr{K}_{1}(t) \phi=\int_{0}^{t_{t}} K_{1}(t, s, \sigma) \phi(t)(\sigma) \mathrm{d} \sigma+K_{1}\left(t, s, t_{f}\right) \phi(t)\left(t_{f}\right) \\
\\
+K_{1}(t, s, 0) \phi(t)(0), \quad V \dot{\phi} \in C\left(\bar{T} ; M^{2}(\bar{T} ; H)\right) .
\end{aligned}
$$

From the definition of inner product in $M^{2}(\bar{T} ; H)$, the 1.h.s. of equation (29a) can be written as

$$
\begin{aligned}
& {\left[\frac{\mathrm{d} \mathscr{K}_{1}(t)}{\mathrm{d} t} \phi_{1}, \phi_{2}\right]} \\
& \quad=\left(\int_{0}^{t_{t}} \frac{\partial K_{1}\left(t, t_{f}, \sigma\right)}{\partial t} \phi_{1}(t)(\sigma) \mathrm{d} \sigma+\frac{\partial K_{1}\left(t, t_{f}, 0\right)}{\partial t} \phi_{1}(t)(0)+\frac{\partial K_{1}\left(t, t_{f}, t_{f}\right)}{\partial t} \phi_{1}(t)\left(t_{f}\right), \phi_{2}(t)\left(t_{f}\right)\right) \\
& \quad+\left(\int_{0}^{t^{\prime}} \frac{\partial K_{1}(t, 0, \sigma)}{\partial t} \phi_{1}(t)(\sigma) \mathrm{d} \sigma+\frac{\partial K_{1}(t, 0,0)}{\partial t} \phi_{1}(t)(0)+\frac{\partial K_{1}\left(t, 0, t_{f}\right)}{\partial t} \phi_{1}(t)\left(t_{f}\right), \phi_{2}(t)(0)\right) \\
& \quad+\int_{0}^{t_{1}}\left(\int_{0}^{t_{1}, \partial K_{1}(t, s, \sigma)} \frac{\partial K_{1}(t, s, 0)}{\partial t} \phi_{1}(t)(\sigma) \mathrm{d} \sigma+\frac{\partial(t)(0)}{\partial t} \phi_{1}\right. \\
& \left.\quad+\frac{\partial K_{1}\left(t, s, t_{f}\right)}{\partial t} \phi_{1}(t)\left(t_{f}\right), \phi_{2}(t)(s)\right) \mathrm{d} s .
\end{aligned}
$$

We find, therefore, that equation (29a) can be represented by nine differential equations. However. we can readily derive the following equalities:

$$
\left.\begin{array}{l}
K_{1}(t, s, 0)=K_{1}^{*}(t, 0, s) \\
K_{1}\left(t, s, t_{f}\right)=K_{1}^{*}\left(t, t_{f}, s\right) \\
K_{1}\left(t, 0, t_{f}\right)=K_{1}^{*}\left(t, t_{f}, 0\right)
\end{array}\right\} \text { for } \begin{aligned}
& 0 \leqslant t \leqslant t_{f} \\
& 0 \leqslant s \leqslant t_{f}
\end{aligned}
$$

where "*" means that for $\phi_{1}, \phi_{2} \in H$ and for fixed $t, s$,

$$
\left(K_{1}^{*} \phi_{1}, \phi_{2}\right)=\left(\phi_{1}, K_{1} \phi_{2}\right) \text {. }
$$

Hence, we need to obtain explicitly only six of these differential equations. Now, we list representations of all terms of the r.h.s. of (29a):

$$
\begin{aligned}
& {\left[\left\langle A I_{1} \phi_{1}, \mathscr{K}_{1}^{*}(t) \phi_{2}\right\rangle\right]=\left\langle A \phi_{1}(t)\left(t_{f}\right) \cdot \int_{0}^{t_{t}} K_{1}^{*}\left(t, s, t_{f}\right) \phi_{2}(t)(s) \mathrm{d} s\right.} \\
& \left.+K_{1}^{*}\left(t, t_{f}, t_{f}\right) \phi_{2}(t)\left(t_{f}\right)+K_{1}^{*}\left(t, 0, t_{j}\right) \phi_{2}(t)(0)\right\rangle \\
& +\int_{1 "}^{t_{t}}\left\langle A I_{\sigma t} \phi_{1}(t)(\sigma), \int_{0}^{t_{1}} K_{1}^{*}(t, s, \sigma) \phi_{2}(t)(s) \mathrm{d} s+K_{1}^{*}\left(t, t_{f}, \sigma\right) \phi_{2}(t)\left(t_{f}\right)\right. \\
& \left.+K_{1}^{*}(t, 0, \sigma) \phi_{1}(t)(0)\right\rangle \mathrm{d} \sigma,
\end{aligned}
$$




$$
\begin{aligned}
& {\left[\left\langle A I_{\cdot}, \phi_{2}, \mathscr{K}_{1}^{*}(t) \phi_{1}\right\rangle\right]=\left\langle A \phi_{2}(t)\left(t_{f}\right), \int_{0}^{t_{f}} K_{1}\left(t, t_{f}, \sigma\right) \phi_{1}(t)(\sigma) \mathrm{d} \sigma\right.} \\
& \left.+K_{1}\left(t, t_{f}, t_{f}\right) \phi_{1}(t)\left(t_{f}\right)+K_{1}\left(t, t_{f}, 0\right) \phi_{1}(t)(0)\right\rangle \\
& +\int_{0}^{t_{t}}\left\langle A I_{s t} \phi_{2}(t)(s), \int_{0}^{t_{f}} K_{1}(t, s, \sigma) \phi_{1}(t)(\sigma) \mathrm{d} \sigma+K_{1}\left(t, s, t_{f}\right) \phi_{1}(t)\left(t_{f}\right)\right. \\
& \left.+K_{1}(t, s, 0) \phi_{1}(t)(0)\right\rangle \mathrm{d} s
\end{aligned}
$$

$$
\begin{aligned}
& {\left[B_{\mathrm{c}} I_{,}, \int_{0}^{t} N_{\mathrm{e}}(t, \sigma) C_{\mathrm{e}} \phi_{1}(t)(\sigma) \mathrm{d} \sigma, \mathscr{K}_{1}^{*}(t) \phi_{2}\right]} \\
& =\int_{0}^{t_{f}}\left(B_{\mathrm{e}} I_{t \sigma} N_{\mathrm{e}}(t, \sigma) C_{\mathrm{e}} \phi_{1}(t)(\sigma), \int_{0}^{t_{f}}\left\{K_{1}^{*}\left(t, s, f_{f}\right)+\int_{t}^{t_{f}} K_{1}^{*}(t, s, \tau) \mathrm{d} \tau\right\} \phi_{2}(t)(s) \mathrm{d} s\right) \mathrm{d} \sigma \\
& \quad+\left(\int_{0}^{t_{f}} B_{\mathrm{e}} I_{t \sigma} N_{\mathrm{e}}(t, \sigma) C_{\mathrm{e}} \phi_{1}(t)(\sigma) \mathrm{d} \sigma,\left\{\left(K_{1}^{*}\left(t, t_{f}, t_{f}\right)+\int_{t}^{t_{f}} K_{1}^{*}\left(t, t_{f}, \tau\right) \mathrm{d} \tau\right) \phi_{2}(t)\left(t_{f}\right)\right.\right. \\
& \left.\left.\quad+\left(K_{1}^{*}\left(t, 0, t_{f}\right)+\int_{t}^{t_{t}} K_{1}^{*}(t, 0, \tau) \mathrm{d} \tau\right) \phi_{2}(t)(0)\right\}\right),
\end{aligned}
$$$$
\left[\mathrm{B}_{\mathrm{e}} I_{t} \int_{0}^{t} N_{\mathrm{e}}(t, \sigma) C_{\mathrm{e}} \phi_{2}(t)(\sigma) \mathrm{d} \sigma, \mathscr{K}_{1}(t) \phi_{1}\right]
$$$$
=\int_{0}^{t_{t}}\left(B_{\mathrm{c}} I_{t s} N_{\mathrm{c}}(t, s) C_{\mathrm{e}} \phi_{2}(t)(s), \int_{0}^{t_{f}}\left\{K_{1}\left(t, t_{f}, \sigma\right)+\int_{1}^{t_{f}} K_{1}(t, \tau, \sigma) \mathrm{d} \tau\right\} \phi_{1}(t)(\sigma) \mathrm{d} \sigma\right) \mathrm{d} s
$$$$
+\left(\int_{0}^{t_{f}} B_{\mathrm{e}} I_{t s} N_{\mathrm{e}}(t, s) C_{\mathrm{e}} \phi_{2}(t)(s) \mathrm{d} s,\left\{\left(K_{1}\left(t, t_{f}, t_{f}\right)+\int_{t}^{t_{f}} K_{1}\left(t, \tau, t_{f}\right) \mathrm{d} \tau\right) \phi_{1}(t)\left(t_{f}\right)\right.\right.
$$$$
\left.\left.+\left(K_{1}\left(t, t_{f}, 0\right)+\int_{t}^{t_{f}} K_{1}(t, \tau, 0) \mathrm{d} \tau\right) \phi_{1}(t)(0)\right\}\right),
$$

$$
\begin{aligned}
& {\left[B_{\mathrm{p}} I_{f}\left(R_{\mathrm{p}}^{*} R_{\mathrm{p}}\right)^{-1} B_{\mathrm{p}}^{*}\left\{\mathscr{K}_{1}(t)\left(t_{f}\right) \phi_{1}+\int_{0}^{t_{f}} \mathscr{K}_{1}(t)(\sigma) \phi_{1} \mathrm{~d} \sigma\right\}, \mathscr{K}_{1}^{*}(t) \phi_{2}\right]} \\
& =\left(\left(R_{\mathrm{p}}^{*} R_{\mathrm{p}}\right)^{-1} \times B_{\mathrm{p}}^{*}\left\{\int_{0}^{t_{f}}\left(K_{1}\left(t, t_{f}, \sigma\right)+\int_{t}^{t_{f}} K_{1}(t, \tau, \sigma) \mathrm{d} \tau\right) \phi_{1}(t)(\sigma) \mathrm{d} \sigma\right.\right. \\
& \left.\quad+\left(K_{1}\left(t, t_{f}, t_{f}\right)+\int_{t}^{t_{f}} K_{1}\left(t, \tau, t_{f}\right) \mathrm{d} \tau\right) \phi_{1}(t)\left(t_{f}\right)+\left(K_{1}\left(t, t_{f}, 0\right)+\int_{t}^{t_{t}} K_{1}(t, \tau, 0) \mathrm{d} \tau\right) \phi_{1}(t)(0)\right\}, \\
& \\
& B_{\mathrm{p}}^{*}\left\{\int_{0}^{t_{f}}\left(K_{1}^{*}\left(t, s, t_{f}\right)+\int_{t}^{t_{t}} K_{1}^{*}(t, s, \tau) \mathrm{d} \tau\right) \phi_{2}(t)(s) \mathrm{d} s+\left(K_{1}^{*}\left(t, t_{f}, t_{f}\right)+\int_{t}^{t_{t}} K_{1}^{*}\left(t, t_{f}, \tau\right) \mathrm{d} \tau\right)\right. \\
& \left.\left.\quad \times \phi_{2}(t)\left(t_{f}\right)+\left(K_{1}^{*}\left(t, 0, t_{f}\right)+\int_{0}^{t_{t}} K_{1}^{*}(t, 0, \tau) \mathrm{d} \tau\right) \phi_{2}(t)(0)\right\}\right)_{H_{u}},
\end{aligned}
$$

$$
\left[\chi \phi_{2}, \chi \phi_{1}\right]=\left[I_{t_{1}} Q \phi_{2}, I_{I_{1}} Q \phi_{1}\right]=\left(\phi_{2}(t)\left(t_{f}\right), Q^{*} Q \phi_{1}(t)\left(t_{f}\right)\right)
$$

and

$$
\begin{aligned}
\left(U \phi_{2}, U \phi_{1}\right)_{H_{u}}=\left(R_{\mathrm{e}} \int_{0}^{t} N_{\mathrm{e}}(t, s) C_{\mathrm{e}} \phi_{2}(t)(s) \mathrm{d} s, R_{\mathrm{e}} \int_{0}^{t} N_{\mathrm{e}}(t, \sigma) C_{\mathrm{e}} \phi_{1}(t)(\sigma) \mathrm{d} \sigma\right)_{H_{u}} \\
=\int_{0}^{t_{f}} \int_{0}^{t_{\zeta}}\left(\phi_{1}(t)(\sigma), I_{t \sigma} C_{\mathrm{e}}^{*} N_{\mathrm{e}}^{*}(t, \sigma) R_{\mathrm{e}}^{*} R_{\mathrm{e}} N_{\mathrm{e}}(t, s) C_{\mathrm{e}} I_{t s} \phi_{2}(t)(s)\right) \mathrm{d} s \mathrm{~d} \sigma .
\end{aligned}
$$


Consequently, from (29a), (44)-(53), we can derive the following equations:

$$
\begin{aligned}
& -\left(\frac{\partial K_{1}(t, s, \sigma)}{\partial t} \phi_{1}, \phi_{2}\right) \\
& =\left\langle A I_{\sigma t} \phi_{1}, K_{1}^{*}(t, s, \sigma) \phi_{2}\right\rangle+\left\langle A I_{s t} \phi_{2}, K_{1}(t, s, \sigma) \phi_{1}\right\rangle \\
& +\left(B_{\mathrm{c}} I_{t s} N_{\mathrm{e}}(t, s) C_{\mathrm{c}} \phi_{2},\left\{K_{1}\left(t, t_{i}, \sigma\right)+\int_{1}^{t_{t}} K_{1}(t, \tau, \sigma) \mathrm{d} \tau\right\} \phi_{1}\right) \\
& +\left(B_{\mathrm{c}} I_{t \sigma} N_{\mathrm{c}}(t, \sigma) C_{\mathrm{e}} \phi_{1},\left\{K_{1}^{*}\left(t, s, t_{f}\right)+\int_{t}^{t_{\prime}} K_{1}^{*}(t, s, \tau) \mathrm{d} \tau\right\} \phi_{2}\right) \\
& -\left(\left(K_{1}\left(t, t_{f}, \sigma\right)+\int_{i}^{t_{i}} K_{1}(t, \tau, \sigma) \mathrm{d} \tau\right) \phi_{1}, B_{\mathrm{p}}\left(R_{p}^{*} R_{\mathrm{p}}\right)^{-1} B_{\mathrm{p}}^{*}\right. \\
& \left.\times\left(K_{1}^{*}\left(t, s, t_{f}\right)+\int_{t}^{t_{t}} K_{1}^{*}(t, s, \tau) \mathrm{d} \tau\right) \phi_{2}\right)-\left(\phi_{1}, I_{t \sigma} C_{\mathrm{e}}^{*} N_{\mathrm{e}}^{*}(t, \sigma) R_{\mathrm{e}}^{*} R_{\mathrm{c}} N_{\mathrm{e}}(t, s) C_{\mathrm{c}} I_{t s} \phi_{2}\right) \\
& \text { in } 0<t<t_{f}, 0<s<t_{f} \text { and } 0<\sigma<t_{f}, \quad \forall \phi_{1}, \phi_{2} \in V \\
& \left(K_{1}\left(t_{f}, s, \sigma\right) \phi_{1}, \sigma_{2}\right)=0 \quad \text { on } 0<s<t_{f} \text { and } 0<\sigma<t_{f} \\
& -\left(\frac{\partial K_{1}(t, s, 0)}{\partial t} \phi_{1}, \phi_{2}\right) \\
& =\left\langle A I_{s t} \phi_{2}, K_{\mathrm{l}}(t, s, 0) \phi_{1}\right\rangle+\left(B_{\mathrm{e}} I_{t s} N_{\mathrm{e}}(t, s) C_{\mathrm{c}} \phi_{2},\left(K_{1}\left(t, t_{f}, 0\right)+\int_{t}^{t_{f}} K_{1}(t, \tau, 0) \mathrm{d} \tau\right) \phi_{1}\right) \\
& -\left(\left(K_{1}\left(t, t_{f}, 0\right)+\int_{t}^{t_{t}} K_{1}(t, \tau, 0) \mathrm{d} \tau\right) \phi_{1},\right. \\
& \left.B_{\mathrm{p}}\left(R_{\mathrm{p}}^{*} R_{\mathrm{p}}\right)^{-1} B_{\mathrm{p}}^{*}\left(K_{1}\left(t, s, t_{f}\right)+\int_{t}^{t_{f}} K_{1}^{*}(t, s, \tau) \mathrm{d} \tau\right) \phi_{2}\right) \\
& \text { in } 0<t<t_{f} \text { and } 0<s<t_{f}, \quad \forall \phi_{1}, \phi_{2} \in V \\
& \left(K_{1}\left(t_{f}, s, 0\right) \phi_{1}, \phi_{2}\right)=0 \quad \text { on } 0<s<t_{f} \text { with } K_{1}(t, s, 0)=K_{1}^{*}(t, 0, s) \text {, } \\
& -\left(\frac{\partial K_{1}\left(t, s, t_{f}\right)}{\partial t} \phi_{1}, \phi_{2}\right) \\
& =\left\langle A I_{s t} \phi_{2}, K_{1}\left(t, s, t_{f}\right) \phi_{1}\right\rangle+\left\langle A \phi_{1}, K_{1}^{*}\left(t, s, t_{f}\right) \phi_{2}\right\rangle \\
& +\left(B_{\mathrm{e}} I_{t s} N_{\mathrm{c}}(t, s) C_{\mathrm{c}} \phi_{2},\left(K_{1}\left(t, t_{f}, t_{j}\right)+\int_{t}^{t_{f}} K_{1}\left(t, \tau, t_{f}\right) \mathrm{d} \tau\right) \phi_{1}\right) \\
& -\left(\left(K_{1}\left(t, t_{f}, t_{f}\right)+\int_{t}^{t_{f}} K_{1}\left(t, \tau, t_{f}\right) \mathrm{d} \tau\right) \phi_{1},\right. \\
& \left.B_{\mathrm{p}}\left(R_{\mathrm{p}}^{*} R_{\mathrm{p}}\right)^{-1} B_{\mathrm{p}}^{*}\left(K_{1}^{*}\left(t, s, t_{f}\right)+\int_{t}^{t_{f}} K_{1}^{*}(t, s, \tau) \mathrm{d} \tau\right) \phi_{2}\right) \\
& \text { in } 0<t<t_{f} \text { and } 0<s<t_{f} \quad \forall \phi_{1}, \phi_{2} \in V \\
& \left(K_{1}\left(t_{f}, s, t_{f}\right) \phi_{1}, \phi_{2}\right)=0 \quad \text { in } 0<s<t_{f} \text { with } K_{1}\left(t, s, t_{f}\right)=K_{1}^{*}\left(t, t_{f}, s\right) \\
& \int-\left(\frac{\partial K_{1}\left(t, 0, t_{f}\right)}{\partial t} \phi_{1}, \phi_{2}\right) \\
& =\left\langle A \phi_{1}, K_{1}^{*}\left(t, 0, t_{f}\right) \phi_{2}\right\rangle-\left(\left(K_{1}\left(t, t_{f}, t_{f}\right)+\int_{1}^{t_{1}} K_{1}\left(t, \tau, t_{f}\right) \mathrm{d} \tau\right) \phi_{1},\right. \\
& \left.B_{\mathrm{p}}\left(R_{\mathrm{p}}^{*} R_{\mathrm{p}}\right)^{-1} B_{\mathrm{p}}^{*}\left(K_{1}^{*}\left(t, 0, t_{j}\right)+\int_{t}^{t_{t}} K_{1}^{*}(t, 0, \tau) \mathrm{d} \tau\right) \phi_{2}\right) \quad \text { in } \quad 0<t<t_{f} \quad \forall \phi_{1}, \phi_{2} \in V \\
& \left(K_{1}\left(t_{f}, 0, t_{f}\right) \phi_{1}, \phi_{2}\right)=0 \quad \text { with } \quad K_{1}\left(t, 0, t_{f}\right)=K_{1}^{*}\left(t, t_{f}, 0\right)
\end{aligned}
$$




$$
\left\{\begin{array}{l}
-\left(\frac{\partial K_{1}(t, 0,0)}{\partial t} \phi_{1}, \phi_{2}\right) \\
=-\left(\left(K_{1}\left(t, t_{f}, 0\right)+\int_{t}^{t_{f}} K_{1}(t, \tau, 0) \mathrm{d} \tau\right) \phi_{1},\right. \\
\left.B_{\mathrm{p}}\left(R_{\mathrm{p}}^{*} R_{\mathrm{p}}\right)^{-1} B_{\mathrm{p}}^{*}\left(K_{1}^{*}\left(t, 0, t_{f}\right)+\int_{t}^{t_{f}} K_{1}^{*}(t, 0, \tau) \mathrm{d} \tau\right) \phi_{2}\right) \text { in } 0<t<t_{f} \quad \forall \phi_{1}, \phi_{2} \in V \\
\left(K_{1}\left(t_{f}, 0,0\right) \phi_{1}, \phi_{2}\right)=0
\end{array}\right.
$$

and

$$
\left\{\begin{array}{l}
-\left(\frac{\partial K_{1}\left(t, t_{f}, t_{f}\right)}{\partial t} \phi_{1}, \phi_{2}\right) \\
=\left\langle A \phi_{2}, K_{1}\left(t, t_{f}, t_{f}\right) \phi_{1}\right\rangle+\left\langle A \phi_{1}, K_{1}^{*}\left(t, t_{f} t_{f}\right) \phi_{2}\right\rangle \\
-\left(\left(K_{1}\left(t, t_{f}, t_{f}\right)+\int_{t}^{t_{f}} K_{1}\left(t, \tau, t_{f}\right) \mathrm{d} \tau\right) \phi_{1},\right. \\
\left.B_{\mathrm{p}}\left(R_{\mathrm{p}}^{*} R_{\mathrm{p}}\right)^{-1} B_{\mathrm{p}}^{*}\left(K_{1}\left(t, t_{f}, t_{f}\right)+\int_{t}^{t_{f}} K_{1}^{*}\left(t, t_{f}, \tau\right) \mathrm{d} \tau\right) \phi_{2}\right)+\left(\phi_{2}, Q^{*} Q \phi_{1}\right) \\
\text { in } 0<t<t_{f} \quad \forall \phi_{1}, \phi_{2} \in V \\
\quad\left(K_{1}\left(t_{f}, t_{f}, t_{f}\right) \phi_{1}, \phi_{2}\right)=0 .
\end{array}\right.
$$

By using the similar technique mentioned above, the gain operators $\mathscr{K}_{2}(t), \mathscr{P}_{i}(t)$, and $\hat{\pi}(t), \hat{\eta}_{\mathrm{c}}(t)$ can also be represented by means of the kernel equations.

\section{CONCLUSION}

Under the assumption of the existence of saddle point in the class of linear controls, we have explicitly solved stochastic pursuit-evasion infinite dimensional differential games. As in [1], one can give a sufficient condition for the existence of saddle point for our situation as well.

This condition essentially says that, for $R_{\mathrm{p}}^{-1}$ and $R_{\mathrm{c}}^{-1}$ sufficiently small, a saddle point always exists. This is not wholly satisfactory and obtaining a more explicit condition in terms of the system parameters is highly desirable.

\section{REFERENCES}

1. A. Bagchi and G. J. Olsder, Linear-quadratic stochastic pursuit-evasion games. Appl. Math. Optim. 7, 95-123 (1981).

2. W. W. Wilman, Formal solutions for a class of stochastic pursuit-evasion games. IEEE Trans. Automat. Control AC-14, 504.509 (1969).

3. P. R. Kumar and J. H. van Schuppen, On Nash equilibrium solutions in stochastic dynamical games. IEEE Trans. Automat. Control AC-25, 1146-1149 (1980).

4. A. Bagchi and G. J. Olsder, Numerical approaches to linear-quadratic differential games with imperfect observations. J. Franklin Inst. 315, 423-433 (1983).

5. A. Bensoussan, Filtrage Optimal Des Systèmes Linéaires. Dunod, Paris (1971).

6. A. Bensoussan. Control of stochastic partial differential equations. In Distributed Parameter Systems (Edited by W. H. Ray and D. G. Lainiotis). Marcel Dekker, New York (1978).

7. A. Bensoussan and M. Viot, Optimal stochastic control for linear distributed parameter systems. SIAM J. Control 13, $904926(1975)$. 